\title{
Auditory and Visual System White Matter Is Differentially Impacted by Normative Aging in Macaques
}

\author{
Daniel T. Gray, ${ }^{1,2}$ Nicole M. De La Peña, ${ }^{1,2}$ Lavanya Umapathy, ${ }^{3}$ Sara N. Burke, ${ }^{4}{ }^{\circledR}$ James R. Engle, ${ }^{1,2}$ \\ Theodore P. Trouard, ${ }^{2,5}$ and ${ }^{\circledR}$ Carol A. Barnes ${ }^{1,2,6}$ \\ ${ }^{1}$ Division of Neural System, Memory and Aging, University of Arizona, Tucson, Arizona 85724, ${ }^{2}$ Evelyn F. McKnight Brain Institute, University of \\ Arizona, Tucson, Arizona 85724, ${ }^{3}$ Electrical and Computer Engineering, University of Arizona, Tucson, Arizona 85724, ${ }^{4}$ Evelyn F. McKnight Brain \\ Institute, University of Florida, Gainesville, Florida 32609, ${ }^{5}$ Department of Biomedical Engineering, University of Arizona, Tucson, Arizona 85724, \\ and ${ }^{6}$ Departments of Psychology, Neurology and Neuroscience, University of Arizona, Tucson, Arizona 85724
}

Deficits in auditory and visual processing are commonly encountered by older individuals. In addition to the relatively well described age-associated pathologies that reduce sensory processing at the level of the cochlea and eye, multiple changes occur along the ascending auditory and visual pathways that further reduce sensory function in each domain. One fundamental question that remains to be directly addressed is whether the structure and function of the central auditory and visual systems follow similar trajectories across the lifespan or sustain the impacts of brain aging independently. The present study used diffusion magnetic resonance imaging and electrophysiological assessments of auditory and visual system function in adult and aged macaques to better understand how age-related changes in white matter connectivity at multiple levels of each sensory system might impact auditory and visual function. In particular, the fractional anisotropy (FA) of auditory and visual system thalamocortical and interhemispheric corticocortical connections was estimated using probabilistic tractography analyses. Sensory processing and sensory system FA were both reduced in older animals compared with younger adults. Corticocortical FA was significantly reduced only in white matter of the auditory system of aged monkeys, while thalamocortical FA was lower only in visual system white matter of the same animals. Importantly, these structural alterations were significantly associated with sensory function within each domain. Together, these results indicate that age-associated deficits in auditory and visual processing emerge in part from microstructural alterations to specific sensory white matter tracts, and not from general differences in white matter condition across the aging brain.

Key words: auditory brainstem response; diffusion MRI; sensory deficits; splenium; thalamic radiation; visual evoked potential

Significance Statement

Age-associated deficits in sensory processing arise from structural and functional alterations to both peripheral sensory organs and central brain regions. It remains unclear whether different sensory systems undergo similar or distinct trajectories in function across the lifespan. To provide novel insights into this question, this study combines electrophysiological assessments of auditory and visual function with diffusion MRI in aged macaques. The results suggest that age-related sensory processing deficits in part result from factors that impact the condition of specific white matter tracts, and not from general decreases in connectivity between sensory brain regions. Such anatomic specificity argues for a framework aimed at understanding vulnerabilities with relatively local influence and brain region specificity.

Received May 8, 2020; revised Aug. 6, 2020; accepted Oct. 4, 2020.

Author contributions: D.T.G., S.N.B., J.R.E., and C.A.B. designed research; D.T.G. performed research; L.U. and T.P.T. contributed unpublished reagents/analytic tools; D.T.G., N.M.D.L.P., L.U., and T.P.T. analyzed data; D.T.G. wrote the paper.

This work was supported by National Institutes of Health Grants R01-AG-050548 and F31-AG-055263, the McKnight Brain Research Foundation, and the Arizona Department of Health Services. We thank Mike Valdez for design and fabrication of the MRl-compatible nonhuman primate stereotactic frame; Scott Squire for assistance in MRl; Kojo Plange for behavioral training; and Luann Snyder and Michelle Albert for administrative assistance.

The authors declare no competing financial interests.

Correspondence should be addressed to Carol A. Barnes at carol@nsma.arizona.edu.

https://doi.org/10.1523/JNEUROSCI.1163-20.2020

Copyright $\odot 2020$ the authors

\section{Introduction}

Normative brain aging results in decreased function across multiple sensory systems that compromise an older individual's ability to detect and process behaviorally relevant information and can substantially reduce quality of life (Brown and Barrett, 2011; Bourne et al., 2017). Deficits in auditory and visual system function are common in older people (Gopinath et al., 2009; Bourne et al., 2017; Wattamwar et al., 2017), and numerous age-associated pathologies at the level of the cochlea and in the retina reduce the ability of each system to transduce sensory 
information (Ryan, 2000; Schmiedt, 2010; Lim et al., 2012; Gheorghe et al., 2015). Additionally, structural and functional alterations also emerge within central auditory and visual system networks downstream from the cochlea and retina (Husain et al., 2011; Owsley, 2011; Andersen, 2012; Lin et al., 2014). Advances in the quality and precision of sensory prosthetics have made it clear that distinct sensory deficits require unique approaches (Plontke et al., 2017; Farnum and Pelled, 2020). Precisely understanding how particular age-associated neurobiological changes impact different facets of sensory processing is critical for optimizing intervention strategies that maintain sensory function in older individuals.

One major challenge facing research in the field of aging sensory systems is that numerous environmental factors negatively impact sensory-processing abilities. For example, hearing deficits have been linked with exposure to various ototoxic chemicals (Campo et al., 2013) and loud noise stimuli (Fransen et al., 2008); and, similarly, ultraviolet light exposure can negatively impact visual processing (Yam and Kwok, 2014). Furthermore, health conditions such as cardiovascular disease and type 2 diabetes mellitus also increase an individual's likelihood of developing sensory deficits (Mitchell et al., 2009; Helzner et al., 2011). Observations like these make it difficult to decipher whether sensory deficits observed in an older individual are because of normative changes that occur in aging sensory systems or from that person's unique experience. Because environmental variables can be better controlled in animal models, their use can help isolate normative age-associated changes that occur in sensory brain circuits from those that result from exposure to environmental factors. Striking similarities in the organization of sensory and cognitive brain circuits between macaques and humans (Hackett et al., 2001; Petrides and Pandya, 2003) make nonhuman primates a strong animal model for the study of normative brain aging (Hara et al., 2012; Gray and Barnes, 2019).

The central auditory and visual systems of humans and macaques share several basic organizational principles. In both species, the auditory and visual systems form relatively dense reciprocal thalamocortical connections between primary sensory cortices and the ventrolateral posterior thalamus (Jones, 2009; Sherman, 2017). Furthermore, both systems also form interhemispheric corticocortical connections through the posterior extent of the corpus callosum (Essen et al., 1982; Bamiou et al., 2007). Declines in white matter connectivity as assessed using diffusion MRI are hallmark structural changes of normative brain aging (Abe et al., 2008; Bennett et al., 2010). In both humans and macaques, decreases in fractional anisotropy (FA) of the white matter connecting higher-level association areas are thought to reflect functional disconnections that impair an individual's ability to integrate information (Makris et al., 2007; Bennett and Madden, 2014; Lockhart and DeCarli, 2014; Gray et al., 2019). There are far fewer diffusion MRI studies that have investigated age-related structural changes in sensory system white matter, and the data that do exist are conflicting, some reporting FA decreases in sensory white matter across the lifespan and others suggesting that it remains stable (Chang et al., 2004; Madden et al., 2004; Husain et al., 2011; Profant et al., 2014). The principle goals of the present study were to establish in macaque monkeys (1) whether auditory and visual system corticocortical and thalamocortical white matter microstructure is altered across the lifespan, (2) whether the two sensory systems express these age-associated differences similarly or uniquely, and (3) how such changes relate to individual differences in auditory and visual system function.

\section{Materials and Methods}

Animals

Five aged (mean age, 26 years; age range, $24.25-30.8$ years) and six adult (mean age, 13.3 years; age range, 11.25-15 years) female bonnet macaques (Macaca radiata) were used in the present study. Behavioral, electrophysiological, and both structural and diffusion MRI data from nonsensory, higher-level associative frontal and temporal lobe brain structures involved in cognition have been obtained from these animals and reported previously (Burke et al., 2011, 2014; Gray et al., 2017, 2018, 2019; Comrie et al., 2018). The veterinary staff at the University of Arizona (Tucson, AZ) performed semiannual health evaluations on all animals, and no animal included in this study presented with critical health concerns before or during testing. All monkeys were pair housed in a temperature- and humidity-controlled vivarium with a $12 \mathrm{~h}$ light/ dark cycle and ad libitum access to food and water. All experimental protocols described in this study were approved by the Institutional Animal Care and Use Committee at the University of Arizona and followed the guidelines set by the National Institutes of Health.

\section{Auditory and visual sensory measures}

Auditory brainstem response. The auditory brainstem response (ABR) recording protocols followed guidelines published in previous studies that have assessed auditory function using these methods in macaques (Fowler et al., 2010; Engle et al., 2013; Ng et al., 2015). A detailed description of these recording procedures has been published previously (Gray et al., 2019), and are briefly described here.

Monkeys were anesthetized with a mixture of ketamine $(2.0 \mathrm{mg} / \mathrm{kg})$, midazolam $(0.2 \mathrm{mg} / \mathrm{kg})$, and dexmedetomidine $(0.01 \mathrm{mg} / \mathrm{kg})$, and placed in the prone position on a surgical table with their heads elevated. Oxygen saturation levels, heart rates, and respiratory rates were monitored continuously and recorded at minimum every $15 \mathrm{~min}$. Soft insert earphones (ER3A transducers, ETYMOTIC) were placed into each ear canal, and the skin behind each ear, on the forehead, and back were cleaned using an alcohol scrub before 22 gauge stainless steel electrodes were placed subcutaneously at each location (Allen and Starr, 1978; Fowler et al., 2010). Evoked potentials were acquired using Intelligent Hearing Systems (SmartEP Win USB, version 3.97) software on a laptop computer. Pure tones $(2,8,16$, or $32 \mathrm{kHz})$ and click stimuli were used as auditory stimuli. Only ABR peak IV was analyzed since this waveform was the most reliably observed (Ng et al., 2015).

Two different ABRs were collected. The first of these was the threshold ABR, which serves as an estimate of acoustic thresholds. Briefly, auditory stimuli were first presented at an intensity of $80 \mathrm{~dB}$ peak sound pressure level (SPL), and the intensity of these stimuli was reduced in $20 \mathrm{~dB}$ steps until discernable ABR waveforms were no longer apparent. At this point, the SPL of the acoustic stimuli was raised by $5 \mathrm{~dB}$ until the ABR waveforms became evident again to establish the threshold. Note that all evoked potentials were bandpass filtered between 100 and $1500 \mathrm{~Hz}$ to extract the ABR waveforms. A temporal ABR was also collected using the same hardware and software. In this procedure, puretone acoustic stimuli were presented binaurally at $60 \mathrm{~dB}$ SPL, first at a stimulus presentation rate of $50 \mathrm{~Hz}$, and then at $20 \mathrm{~Hz}$. The latency of peak IV was estimated for both the 50 and $20 \mathrm{~Hz}$ conditions by extracting the time from stimulus onset at which the peak amplitude of the wave in consideration occurred. Latency differences between the two conditions $(50-20 \mathrm{~Hz})$ were calculated and used as an estimate of temporal auditory-processing abilities (Mehraei et al., 2016, 2017). Because these evoked potentials reflect the combined activity of a number of pathways up the ascending auditory system, the goal of the present study was to obtain integrated estimates of overall auditory processing, rather than to define the function of a specific, single white matter tract.

Auditory middle latency response. Auditory middle latency response (MLR) thresholds were extracted by presenting pure-tone stimuli $(2,8$, 16 , or $32 \mathrm{kHz}$ ) at sequentially diminishing sound intensities until the $\mathrm{P}_{0}$ component ( $\sim 21-28 \mathrm{~ms}$ ) of the MLR disappeared, similar to the protocol used to determine ABR thresholds. To extract the $\mathrm{P}_{0}$ component, evoked responses were bandpass filtered between 3 and $1500 \mathrm{~Hz}$.

Visual evoked potentials. Visual evoked potentials (VEPs) were acquired using the same Intelligent Hearing Systems software (SmartEP 
Win USB, version 3.97) as was used during the ABR recordings. Electrode placements were directed by the guidelines set by the SmartEP manufacturer, with adjustments to fit the macaque head. Animals were situated in the prone position with their heads elevated. The monkeys received a visual stimulus of a full-field checkerboard pattern delivered by the Intelligent Hearing Systems VEP stimulator placed $\sim 50 \mathrm{~cm}$ in front of the face of the monkey. These stimuli were delivered at either 1 or $2 \mathrm{~Hz}$. Evoked potentials were bandpass filtered at $1-300 \mathrm{~Hz}$. Adult and aged animals reliably showed an evoked potential of $\sim 75 \mathrm{~ms}$, which is referred to as $\mathrm{P}_{75}$. The latency was defined as the time between stimulus onset and the peak amplitude of the $\mathrm{P}_{75}$. The latency difference was calculated by subtracting the $\mathrm{P}_{75}$ latency of the $1 \mathrm{~Hz}$ condition from the $2 \mathrm{~Hz}$ condition, and the latency difference between these two conditions $(2-1 \mathrm{~Hz})$ was used as an estimate of information-processing abilities in the visual system. In contrast to ABR measures that include significant contributions from subcortical auditory nuclei, the primary contribution to the VEP signal is cortical, with a lesser contribution from earlier visual pathways. Like the ABR, the VEP signal reflects integrated estimates of overall visual processing rather than the function of a unique pathway.

Composite sensory scores. Data from the threshold-ABR, temporal$\mathrm{ABR}, \mathrm{MLR}$, and VEP were $z$ score normalized to standardize the units of all electrophysiological data. The composite sensory $z$ score for each animal was determined by an average of all of the $z$ scores. Note that lower $z$ scores indicate better sensory function.

\section{Diffusion tensor imaging and tractography}

Image acquisition and processing. The imaging acquisition and processing protocol is described in detail in the study by Gray et al. (2018). Briefly, all monkeys were anesthetized with intramuscular injections containing midazolam $(0.15-0.2 \mathrm{mg} / \mathrm{kg})$, ketamine $(1.5-2.0 \mathrm{mg} / \mathrm{kg})$, and dexmedetomidine $(0.007-0.01 \mathrm{mg} / \mathrm{kg})$, with older animals receiving the lower ends of these dose ranges. After sedation, the monkeys were intubated, and anesthesia was maintained using 2-3\% sevoflurane delivered through an MRI-compatible vaporizer. All magnetic resonance images were obtained on a 3T SIGNA scanner (GE Healthcare), using a body coil for the radio frequency excitation and an eight-channel head coil for reception. Diffusion-weighted (DW) images were obtained using a single-shot echoplanar imaging sequence. The data were acquired over 51 diffusion directions in a HARDI (high angular-resolution diffusion imaging) sampling scheme over a single shell with a b value of 1000 $\mathrm{s} / \mathrm{mm}^{2}$. Note that previous groups have used HARDI sampling schemes to detect the acoustic radiations in human participants (Berman et al., 2013). The acoustic radiations are particularly challenging to detect via tractography because of their relatively small size and their orientation perpendicular to the myelinated axons contained within the longitudinal fasciculi. HARDI sampling is able to detect this pathway more reliably than diffusion tensor imaging (DTI) since it requires the acquisition of $>50$ gradient directions at a high $\mathrm{b}$ value as opposed to the six gradient directions and lower $b$ values obtained in DTI. The higher angular resolution that this provides results in a more accurate representation of water diffusion within a voxel. T2-weighted reference images were acquired for each monkey using a fast spin-echo sequence. Last, high-resolution anatomic whole-brain T1-weighted images were acquired with a 3D inversion recovery-prepped spoiled gradient-echo sequence using the same parameters described in Gray et al. (2018).

DICOM (Digital Imaging and Communications in Medicine) images were converted into NIFTI format, and T1, T2, and DW images were skull stripped using masks drawn in MRIcron (https://www.nitrc.org/ projects/mricron). Eddy current distortions in DW images were corrected using an iterative Gaussian process-based registration in FSL (Andersson and Sotiropoulos, 2015). Distortions from B0-field inhomogeneity were corrected using TORTOISE software by nonlinearly registering the DW images to reference T2 images. Coil inhomogeneity was corrected using N4ITK bias correction software (Tustison et al., 2010), and DW images were denoised using a local principal component-based noise removal algorithm outlined in the study by Manjón et al. (2013). DW images were registered to T1 images using the FSL Automated Segmentation Toolbox followed by the FSL Linear Image Registration Tool and a boundary-based registration algorithm (Greve and Fischl,
2009). FA maps were generated from diffusion tensor fitting of the DW images in the native space of each monkey.

Tractography and fractional anisotropy. Probabilistic streamlines were generated between regions of interest (ROIs) in the native space of each animal (see sections below). Streamlines were generated using a multitensor tractography approach in the FSL Diffusion Toolbox ProbtrackX, as defined in the study by Gray et al. (2018). This analysis outputs a probability map in which the value of each voxel is the weighted probability that the voxel belongs to the distinct anatomic pathway between the two seed regions. To account for the impact of partial volume effects on the FA estimates, T1-weighted images were segmented into gray matter, white matter, and CSF using the FSL Automated Segmentation Toolbox (FAST). This analysis outputs the probability that a given voxel belongs to each class of tissue. Values $>0.3$ were used to threshold and binarize the white matter maps, and the voxels that were considered white matter following this automated classification method were used to extract the FA values from the voxels in the probabilistic streamlines. FA values were then normalized by dividing the sum of the probability-weighted FA values by the sum of the total probability in the map.

Tractography and fractional anisotropy. Probabilistic streamlines were generated between ROIs in the native space of each animal (see sections below). Streamlines were generated using a multitensor tractography approach in the FSL Diffusion Toolbox ProbtrackX, as defined in the study by Gray et al. (2018). The output of the analysis in Gray et al. (2018) is a probability map in which the value of each voxel is the weighted probability that the voxel belongs to the distinct anatomic pathway between the two seed regions. To account for the impact of partial volume effects on the FA estimates, T1-weighted images were segmented into gray matter, white matter, and CSF using FAST. FAST segmentation outputs the probability that a given voxel belongs to each class of tissue. A value of 0.3 was used to threshold and binarize the white matter maps, and the voxels that were considered white matter following this automated classification method were used to extract the FA values from the voxels in the probabilistic streamlines. FA values were then normalized by dividing the sum of the probability-weighted FA values by the sum of the total probability in the map.

Regions of interest and probabilistic streamlines. All regions of interest were drawn on T1-weighted images using MRIcron software. The parameters for drawing each ROI, along with the protocol for obtaining the commissural and thalamocortical fiber streamlines, are outlined below.

Superior temporal gyrus ROI and interhemispheric auditory projections. The posterior boundary of the superior temporal gyrus was defined as the MRI section in which the lateral fissure first became visible roughly at the junction of the parietal and temporal lobes. The anterior boundary was defined as the section just posterior to the appearance of the anterior commissure. The ROI was drawn in each section starting from the medial junction between the superior temporal gyrus and the insula. This volume encompasses both the dorsal and lateral banks of the superior temporal gyrus, which includes both the primary and secondary auditory fields in the macaque (Hackett et al., 1998, 2001; Kaas and Hackett, 2000). Two inclusion masks were drawn encompassing the corpus callosum in the sagittal sections just lateral to the midline in both hemispheres, and an exclusion mask was drawn in the coronal section just rostral to the anterior edge of the superior temporal gyri seed ROIs.

Auditory cortex, medial geniculate thalamic nucleus, and acoustic radiation. The auditory cortex ROI was restricted to encompass only the dorsal bank of the superior temporal gyrus, within the lateral fissure. The anterior border was set to the section containing the anterior-most portion of the thalamus, and the posterior border was set as the first section to contain insular cortex, medial to the dorsal bank of the superior temporal gyrus. For the medial geniculate nucleus ROI, the anterior border was set as the fourth section anterior to the first section to contain thalamic tissue anterior to the splenium, which was defined as the posterior border of the ROI. The medial geniculate ROI was bounded medially by the lateral extent of the midbrain, which is located just ventral to this nucleus. The lateral border was set by the lateral geniculate nucleus. Streamlines were generated between the medial geniculate nucleus ROI 
and the auditory cortex ROI. Exclusion masks were set to prevent streamlines from entering the inferior parietal gyrus, as well as along the longitudinal fasciculi anterior and posterior to the auditory cortex ROI.

Visual cortex and interhemispheric visual projections via the posterior forceps. The visual cortex ROIs were drawn beginning at the first section where the occipital pole appears and ends at the first coronal plane posterior to the parietooccipital sulcus. An inclusion mask through the midline of the splenium was drawn, and exclusion masks were created in the first coronal plane anterior to the splenium, as well as through the midline of the brain just posterior to the splenium.

Visual cortex, lateral geniculate thalamic nucleus, and optic radiation. The visual cortex ROI was drawn as described above. The anterior border of the lateral geniculate nucleus ROI was defined as the first section to contain the posterior aspect of the putamen, and the posterior border was the first section to contain thalamic tissue anterior to the splenium. The most medial aspect of the parasubiculum of the hippocampal complex, which is located immediately ventral to the lateral geniculate, was used to define the medial border, and the white matter of the external capsule was the lateral border. Streamlines were generated between the lateral geniculate nucleus ROIs and the visual cortex ROIs. Exclusion masks were set to prevent streamlines from entering the parietal cortex and also along the longitudinal fasciculi to prevent streamlines from being generated anteriorly.

Composite fractional anisotropy estimates. Estimates of FA from each of the sensory white matter tracts analyzed were averaged together for each monkey to produce composite sensory white matter FA values.

\section{Statistical analyses}

Assessment of sensory function. ABR, MLR, and VEP thresholds, latencies, and latency differences across different stimulus frequencies were analyzed with repeated-measures ANOVAs. Analyses of ABR data averaged across stimulus frequencies, as well as MLR and VEP data were analyzed with unpaired $t$ tests. In all cases an $\alpha$ level of 0.05 was used, and $p$ values were Bonferroni-Holm corrected when necessary.

Fractional anisotropy comparisons. FA estimates from each white matter projection were analyzed using repeated-measures ANOVAs with age group (adult and aged) and hemisphere (when applicable) as factors. Post hoc tests were performed in every case using unpaired $t$ tests. Again, an $\alpha$ level of 0.05 was used, and $p$ values were Bonferroni-Holm corrected when necessary.

Regression analyses. The relationships between sensory function and white matter FA were assessed using a robust regression model. This regression method is an alternative to least-squares regression and is commonly used with smaller datasets since it is more resistant to the effects of outliers. In all cases, the significance criterion was $p<0.05$.

\section{Results}

Aged macaques display poorer sensory function

Adult and aged macaques underwent several electrophysiological assessments of auditory and visual system function (Fig. 1A-E). Aged monkeys had significantly higher composite sensory scores than did adult animals (unpaired $t$ test; $n_{\text {adult }}=7 ; n_{\text {aged }}=5$; $p<0.01 ; t_{(10)}=-4.1627$; Fig. $\left.1 F\right)$, indicating poorer sensory function.
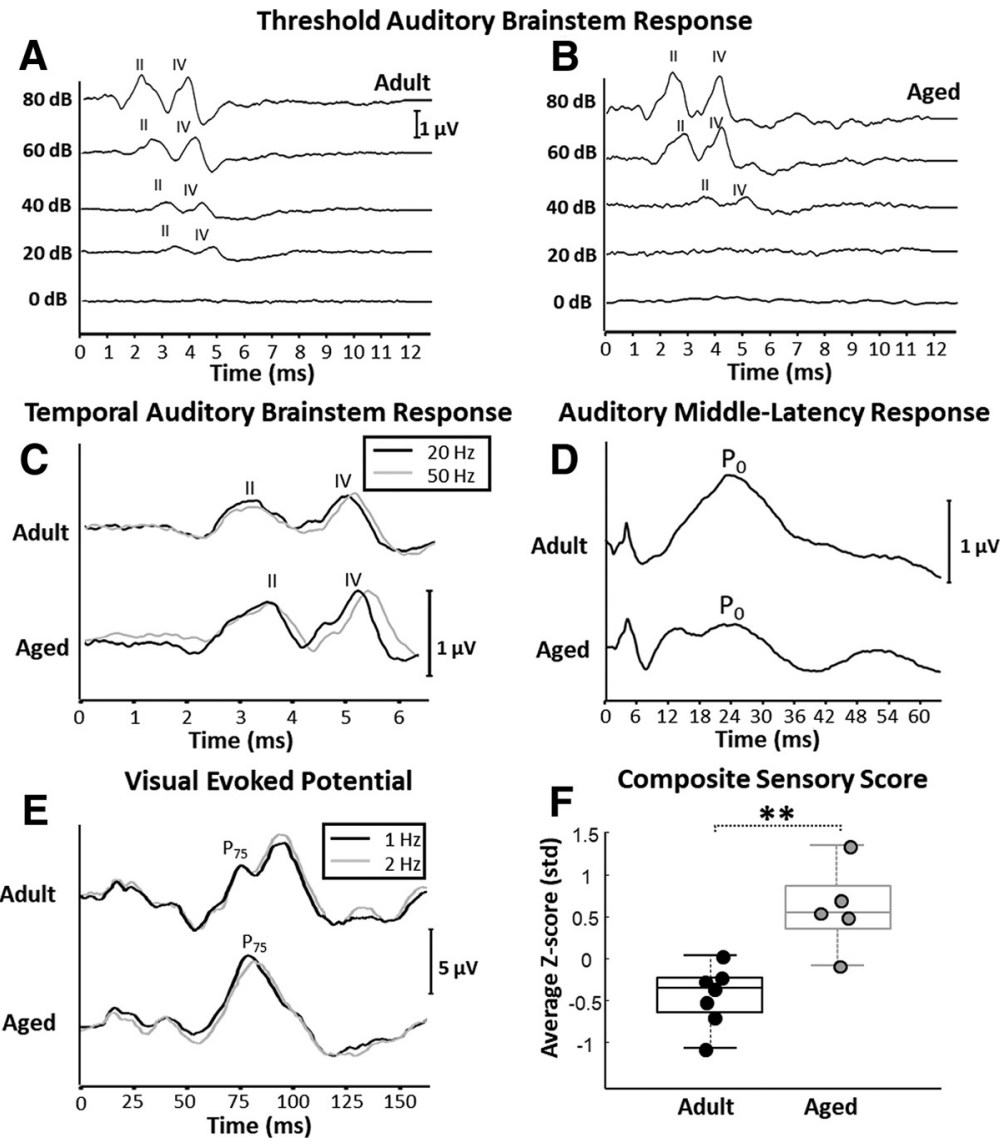

Figure 1. Representative threshold and temporal auditory brainstem response (ABR), auditory middle-latency response (MLR), visual evoked potential (VEP) electrophysiological recordings, and composite sensory scores. $\boldsymbol{A}$, Representative example of an $A B R$ recording from an adult animal used in the threshold $A B R$ analysis. $\boldsymbol{B}$, Representative example of a threshold $A B R$ recording from an aged monkey. In both $\boldsymbol{A}$ and $\boldsymbol{B}$, wave II and wave IV are labeled, and sound presentation occurred at time 0 in both figures. Calibration: $1 \mu \mathrm{V}$. This example shows a lower threshold response $(20 \mathrm{~dB})$ in the adult monkey compared with in the aged monkey $(40 \mathrm{~dB})$. C, Representative temporal-ABR recording from one adult and one aged monkey. The black traces are derived from recordings in which acoustic stimuli were presented at a rate of $20 \mathrm{~Hz}$, whereas gray traces are derived from recordings in which the acoustic stimuli were presented at $50 \mathrm{~Hz}$. The difference in latency of ABR wave IV between the 50 and $20 \mathrm{~Hz}$ conditions was used as an estimate of temporal auditory processing (Mehraei et al., 2016, 2017). Calibration: $1 \mu \mathrm{V}$. Sound presentation occurred at time 0 . Note that the ABR wave IV latency difference between the 20 and $50 \mathrm{~Hz}$ presentation rate conditions appears greater in the aged monkey compared with the adult. $\boldsymbol{D}$, Representative auditory MLR recording from one adult and one aged monkey. Calibration: $1 \mu \mathrm{V}$. Sound presentation occurred at time 0 . Note that the amplitude of the MLR from the aged monkey is smaller than the amplitude of the response of the adult monkey. $\boldsymbol{E}$, Representative examples of one adult and one aged VEP recordings. The black traces are derived from recordings in which visual stimuli were presented at a rate of $1 \mathrm{~Hz}$, whereas gray traces are derived from recordings in which the stimuli were presented at $2 \mathrm{~Hz}$. In both plots, the $P_{75}$ component is labeled. Calibration: $5 \mu \mathrm{V}$. Stimulus presentation occurred at time 0 . As with the temporal ABR, the latency difference of $\mathrm{P}_{75}$ between the 2 and $1 \mathrm{~Hz}$ conditions was used as an estimate of visual system function, and is shown here to be different between the adult and aged monkeys. $\boldsymbol{F}$, Box and whisker plot of composite sensory scores. Boxes denote the middle $50 \%$ of the data, and horizontal lines indicate the median of each distribution. Filled circles represent an individual monkey, with adult animals represented by black circles and aged animals with gray. Aged monkeys had significantly higher composite sensory scores than adult animals. Note that higher sensory scores corresponds with poorer sensory function. ${ }^{* *} p<0.01$.

\section{Results from individual electrophysiological assessments of auditory and visual function}

Auditory brainstem response thresholds

ABR thresholds (Fig. $1 A, B$ ) were measured in each animal using both pure-tone and acoustic click stimuli. Aged macaques displayed higher ABR thresholds (ANOVA; $n_{\text {adult }}=7 ; n_{\text {aged }}=4$; $F_{(1,30)}=8.69, p<0.01$; Fig. $\left.2 A\right)$, and ABR thresholds increased as a function of pure-tone frequency in both age groups (ANOVA; $n_{\text {adult }}=7 ; n_{\text {aged }}=4 ; F_{(3,30)}=15.11, p<0.0001 ;$ Fig. $\left.2 A\right)$. There 

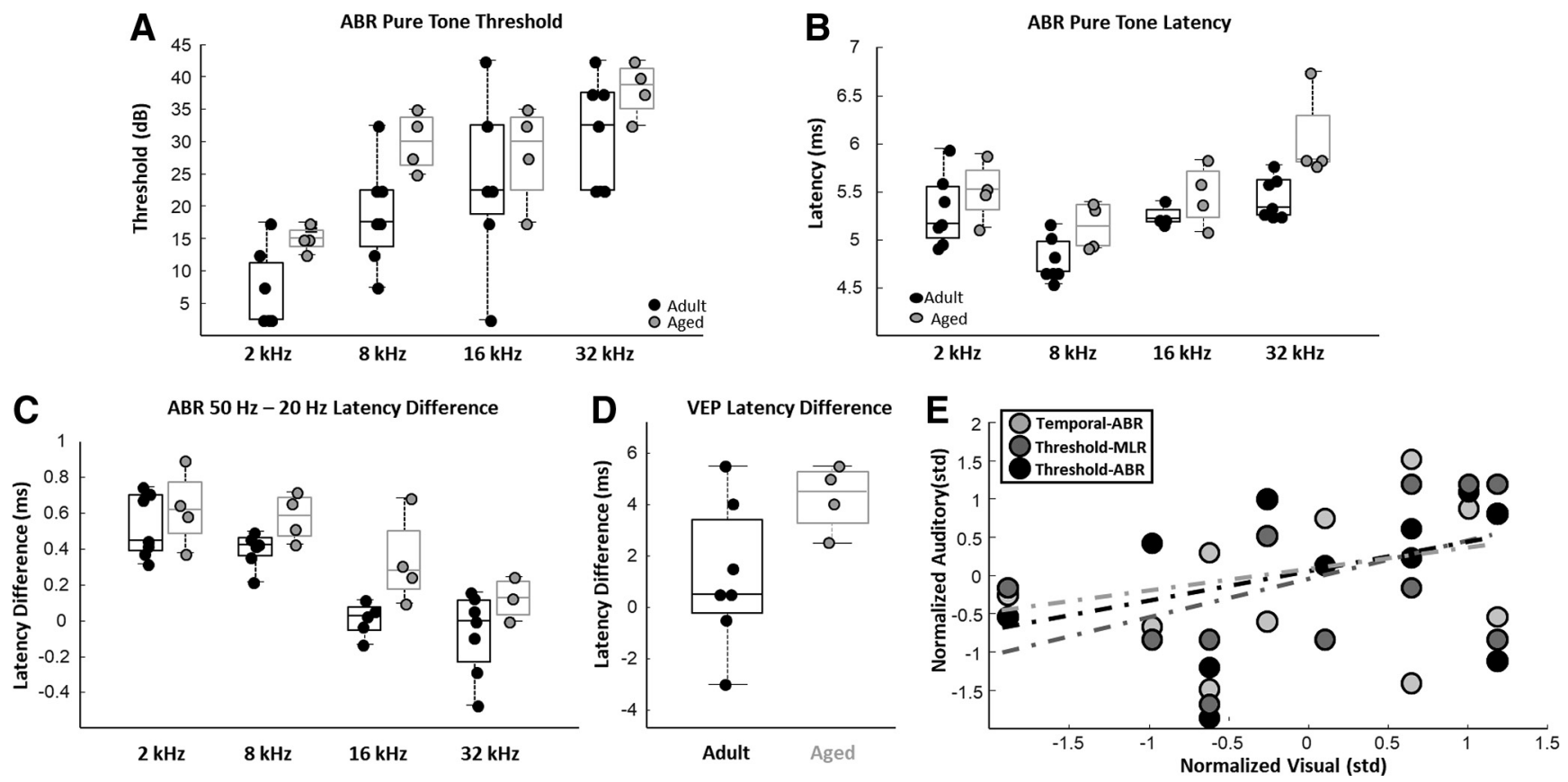

Figure 2. Age comparisons of auditory brainstem response (ABR) and middle-latency response (MLR) thresholds, ABR latencies, temporal-ABR latency differences, and visual evoked potential (VEP) latency differences. A, Box and whisker plots of ABR thresholds across all tested pure-tone frequencies. Boxes denote the middle $50 \%$ of the data, and horizontal lines indicate the median of each distribution. Filled circles represent an individual monkey, with adult animals represented by black circles, and aged animals by gray circles. Aged monkeys had significantly higher ABR thresholds across frequencies compared with the adults. $\boldsymbol{B}$, Box and whisker plots of ABR wave IV latencies across all tested pure-tone frequencies. Box and whisker plot as in $\boldsymbol{A}$. Aged monkeys had significantly longer ABR wave IV latencies across frequencies compared with the adults. $C$, Box and whisker plot of the estimates of temporal acoustic information processing (temporalABR wave IV latency differences) across all tested pure-tone frequencies. Box and whisker plot as in $\boldsymbol{A}$. Aged monkeys had significantly longer latency differences compared with adult animals across all tested pure-tone frequencies. $\boldsymbol{D}$, Box and whisker plot of VEP $P_{75}$ latency differences between the 1 and $2 \mathrm{~Hz}$ stimulus presentation conditions. Box and whisker plot as in $\boldsymbol{A}$. There was no age-related difference in VEP latency differences. $E$, Scatter plot of $z$ score normalized measures of auditory processing (temporal ABR), auditory thresholds (threshold ABR), and auditory middle latency response thresholds plotted against normalized visual system processing (temporal VEP) scores. Dotted trend lines indicate nonsignificant relationships. Visual system function was not significantly associated with any measure of auditory function. ${ }^{*} p<0.05 ;{ }^{* *} p<0.01$.

was no age-by-frequency interaction on $\mathrm{ABR}$ thresholds $\left(\right.$ ANOVA; $\left.n_{\text {adult }}=7 ; n_{\text {aged }}=4 ; F_{(3,30)}=0.39, p=0.76\right)$. ABR thresholds from all tested tone frequencies were averaged together to create a pure tone average (PTA) threshold. PTA thresholds were not statistically different between adult and aged macaques, although aged monkeys tended to have higher thresholds (unpaired $t$ test; $n_{\text {adult }}=7 ; \mathrm{n}_{\text {aged }}=4 ; p=0.07 ; t_{(9)}=-1.99$ ). ABR click thresholds were also not statistically different between adult and aged macaques (unpaired $t$ test; $n_{\text {adult }}=7 ; n_{\text {aged }}=5$; $p=0.12 ; t_{(10)}=-1.67$; data not shown). Likewise, auditory MLR thresholds were not different between age groups (unpaired $t$ test; $\left.n_{\text {adult }}=7 ; n_{\text {aged }}=5 ; p=0.19 ; t_{(10)}=-1.39\right)$.

\section{Auditory brainstem response latencies}

$\mathrm{ABR}$ wave IV latencies to pure-tone stimuli were also measured in each monkey. Wave IV latencies were significantly longer in aged monkeys compared with adults (ANOVA; $n_{\text {adult }}=7 ; n_{\text {aged }}=4$; $F_{(1,30)}=13.23 ; p<0.01$; Fig. $\left.2 B\right)$, and there was also an effect of frequency on $\mathrm{ABR}$ wave IV latencies (ANOVA; $n_{\text {adult }}=7 ; n_{\text {aged }}=4$; $F_{(3,30)}=11.73 ; p<0.01$; Fig. $\left.2 B\right)$. There was no age-by-frequency interaction on $\mathrm{ABR}$ wave IV latencies (ANOVA; $n_{\text {adult }}=7 ; n_{\text {aged }}=$ $\left.4 ; F_{(3,30)}=0.95 ; p=0.43\right)$. PTA latencies were significantly longer in the aged monkeys compared with the adults (unpaired $t$ test; $\left.n_{\text {adult }}=7 ; n_{\text {aged }}=4 ; p<0.05 ; t_{(9)}=-2.35\right)$. Auditory MLR latencies, however, were not different between adult and aged monkeys (unpaired $t$ test; $n_{\text {adult }}=7 ; n_{\text {aged }}=5 ; p=0.41 ; t_{(10)}=-0.86$ ).

Auditory brainstem response estimates of temporal acoustic information processing

In the temporal-ABR analysis (Fig. 1C), pure-tone acoustic stimuli were played to the animals at presentation rates of both 20 and $50 \mathrm{~Hz}$, and the latency difference $(50 \mathrm{~Hz}-20 \mathrm{~Hz})$ between these two conditions was used as an estimate of auditory temporal processing abilities (Mehraei et al., 2016, 2017). Wave IV latency differences between the 50 and $20 \mathrm{~Hz}$ stimulus presentation rate conditions were reduced with increasing tone frequencies in both age groups (ANOVA; $n_{\text {adult }}=7 ; n_{\text {aged }}=4$; $F_{(3,30)}=19.8, p<0.0001$; Fig. $\left.2 C\right)$. Wave IV latency differences were significantly greater in aged monkeys compared with adults $\left(\right.$ ANOVA; $n_{\text {adult }}=7 ; n_{\text {aged }}=4 ; F_{(1,30)}=11.97, p=0.0016$; Fig. $\left.2 C\right)$. There was no interaction between stimulus frequency and age (ANOVA; $\left.F_{(3,30)}=0.66, p=0.58\right)$. PTA latencies were significantly longer in the $50 \mathrm{~Hz}$ presentation rate condition compared with the $20 \mathrm{~Hz}$ condition (ANOVA; $n_{\text {adult }}=7 ; n_{\text {aged }}=4$; $\left.F_{(1,14)}=17.49 ; p<0.01\right)$, and latencies were longer in the aged monkeys compared with the adults in both conditions (ANOVA; $\left.n_{\text {adult }}=7 ; n_{\text {aged }}=4 ; F_{(1,14)}=30.19 ; p<0.01\right)$. There was no ageby-presentation rate interaction on PTA latencies, however (ANOVA; $\left.n_{\text {adult }}=7 ; n_{\text {aged }}=4 ; F_{(1,14)}=2.54 ; p=0.13\right)$. PTA latency differences between these two presentation rate conditions were significantly greater in the aged monkeys compared with the adult animals (unpaired $t$ test; $n_{\text {adult }}=7 ; n_{\text {aged }}=4 ; p<0.01$; $t_{(9)}=-4.68$ ), indicating poorer temporal integration of acoustic information in the older monkeys.

\section{Visual evoked potentials}

In the temporal VEP analysis (Fig. $1 E$ ), visual checkered patterns were given to the animals at presentation rates of either 1 or $2 \mathrm{~Hz}$, and the latency difference between these two conditions $(2 \mathrm{~Hz}-1 \mathrm{~Hz})$ was used as an estimate of visual processing capacities. VEP $\mathrm{P}_{75}$ latencies from stimulus onset were not different 
between adult and aged animals in either the 1 or $2 \mathrm{~Hz}$ stimulus presentation rate conditions (ANOVA; frequency: $F_{(1,11)}=0.48$, $p=0.50$, age: $\left.F_{(1,11)}=0.68, p=0.42\right)$. VEP $\mathrm{P}_{75}$ latency differences between 1 and $2 \mathrm{~Hz}$ stimulus presentation rate conditions were also not statistically different between adult and aged monkeys, although there was a trend toward greater latency differences in aged animals ( $t$ test, $p=0.077, t=-1.99$; Fig. $2 D$ ).

\section{Relationship between electrophysiological estimates of} auditory and visual system function

Relationships between the three measures of auditory sensory function and VEP $\mathrm{P}_{75}$ latency differences were assessed. No measure of auditory function was significantly associated with VEP $\mathrm{P}_{75}$ latency differences, although there were subtle positive trends observed in all three relationships (robust regression; temporalABR: $p=0.36, r=0.34, t=0.97$; threshold-ABR: $p=0.31, r=0.35$, $t=1.08$; threshold-MLR: $p=0.17, r=0.48, t=1.49$; Fig. $2 E)$. This result suggests that age-associated changes in auditory and visual system function arise with some level of independence in aging macaques.

\section{Aged macaques have lower fractional anisotropy in sensory} system white matter

Structural and diffusion MRIs (Fig. 3A) were obtained from all but one monkey that participated in the electrophysiological assessments of auditory and visual system function (Fig. 1). Using probabilistic tractography approaches, FA estimates from thalamocortical and commissural corticocortical white matter tracts from the auditory and visual systems were derived (Fig. $3 B$ ). Compared with adult monkeys, aged animals had significantly lower global sensory FA estimates (unpaired $t$ test; $n_{\text {adult }}=$ $6 ; n_{\text {aged }}=5 ; p<0.05 ; t_{(9)}=2.62$; Fig. $3 C$ ). Monkeys with greater FA in auditory system white matter had greater visual system white matter FA (robust regression; $p<0.01 ; r=0.49 ; t=4.25$; Fig. $3 D$ ). Note that the correlation between auditory and visual system white matter FA was driven by the adult animals, whereas FA estimates from these white matter tracts showed no relationship in aged monkeys (Fig. 3D).

\section{Commissural auditory, but not acoustic radiation white} matter FA, is reduced in aged monkeys

For each monkey, FA estimates were acquired from the following two distinct auditory system white matter tracts: commissural auditory fibers and the acoustic thalamic radiation. FA estimates in the commissural auditory pathway (Fig. $4 A, B$ ) were significantly lower in the aged macaques compared with adults (unpaired $t$ test; $n_{\text {adult }}=6 ; n_{\text {aged }}=5 ; p<0.01 ; t_{(9)}=4.56$; Fig. $4 C$ ). Monkeys that had the highest FA in the commissural auditory pathway had significantly lower ABR thresholds to both pure tones (robust regression; $n=10 ; p=0.01 ; r=-0.67 ; t_{(9)}=-3.09$; Fig. 4D) and acoustic clicks (robust regression; $n=10 ; p=0.01 ; r$ $=-0.74 t_{(9)}=-3.35$; Fig. $4 E$ ). Monkeys with greater FA in the commissural auditory pathways also had lower MLR thresholds (robust regression; $n=10 ; p=0.03 ; r=-0.68 ; t_{(9)}=-2.48$; Fig. $4 F$ ). Similarly, animals with higher FA in the commissural auditory pathway tended to have lower temporal-ABR wave IV latency differences (better auditory temporal processing abilities), although this trend did not reach statistical significance (robust regression; $n=10 ; p=0.07 ; r=-0.63 ; t_{(9)}=-2.04$; Fig. $4 G$ ). Monkeys with higher FA in the commissural auditory pathway tended to have lower temporal-VEP $\mathrm{P}_{75}$ latency differences, although this trend also did not reach statistical significance

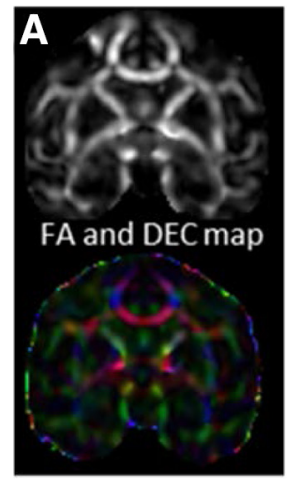

B

Sensory White Matter Analyzed

Acoustic Thalamic Radiation

Commissural Auditory

Optic Thalamic Radiation

Commissural Visual
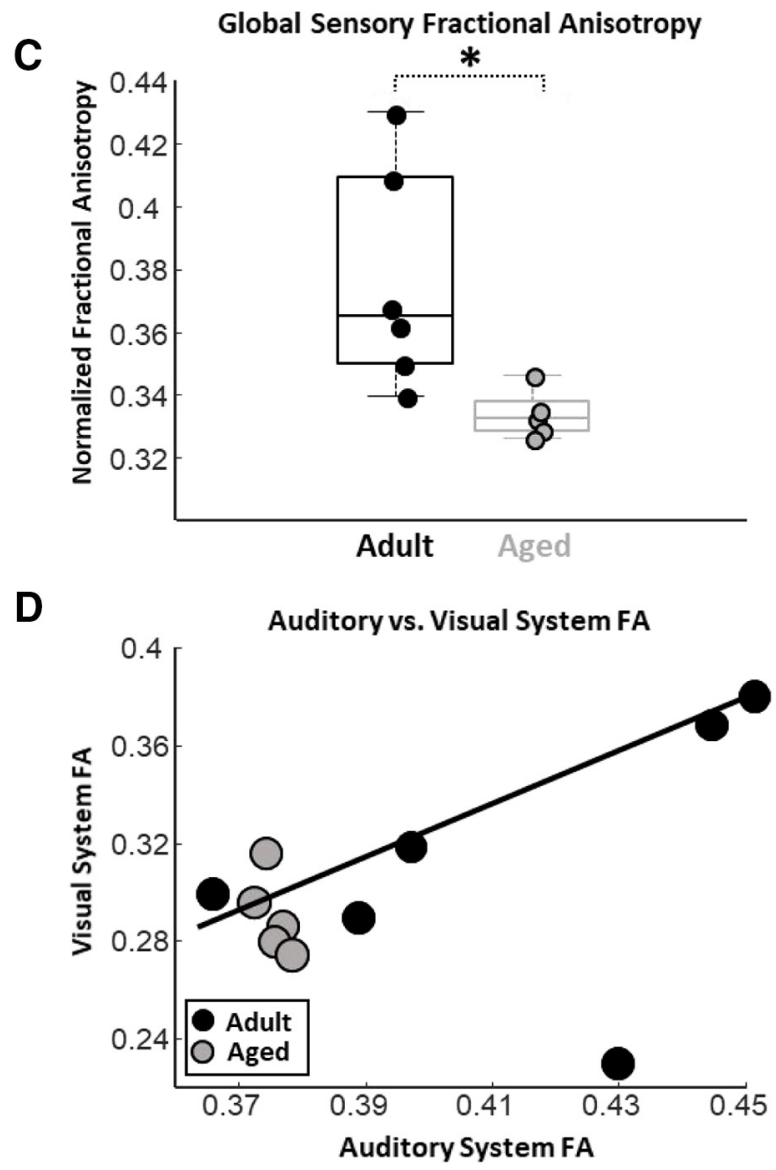

Figure 3. Age comparisons of estimated fractional anisotropy (FA) in auditory and visual system white matter tracts as analyzed by diffusion MRI and probabilistic tractography. A, FA (top) and a directional encoded color (DEC; bottom) map obtained from diffusion-MRI analyses. $\boldsymbol{B}, \mathrm{A}$ list of the auditory and visual system white matter tracts analyzed via probabilistic tractography. C, Box and whisker plot of global sensory FA estimates. Boxes denote the middle $50 \%$ of the data, and horizontal lines indicate the median of each distribution. Filled circles represent an individual monkey, with adult animals represented by black circles, and aged animals by gray circles. Aged monkeys had significantly lower global sensory FA than did adult animals. Note that lower FA is thought to reflect a poorer white matter condition. D, Relationship between auditory system FA and visual system FA. Gray circles represent aged animals, whereas black circles represent adult animals. Solid trend line denotes a significant relationship between auditory system FA and visual system FA. Furthermore, note that there is far less variability in auditory and visual system FA estimates from the aged monkeys compared with the adults. ${ }^{*} p<0.05$.

(robust regression; $n=10 ; p=0.09 ; r=-0.3 ; t_{(9)}=-1.95$; data not shown).

Unlike the commissural auditory pathway, FA estimates derived from the acoustic thalamic radiations (Fig. $4 H, I$ ) were not different between adult and aged macaques (ANOVA; $n_{\text {adult }}=6$; 

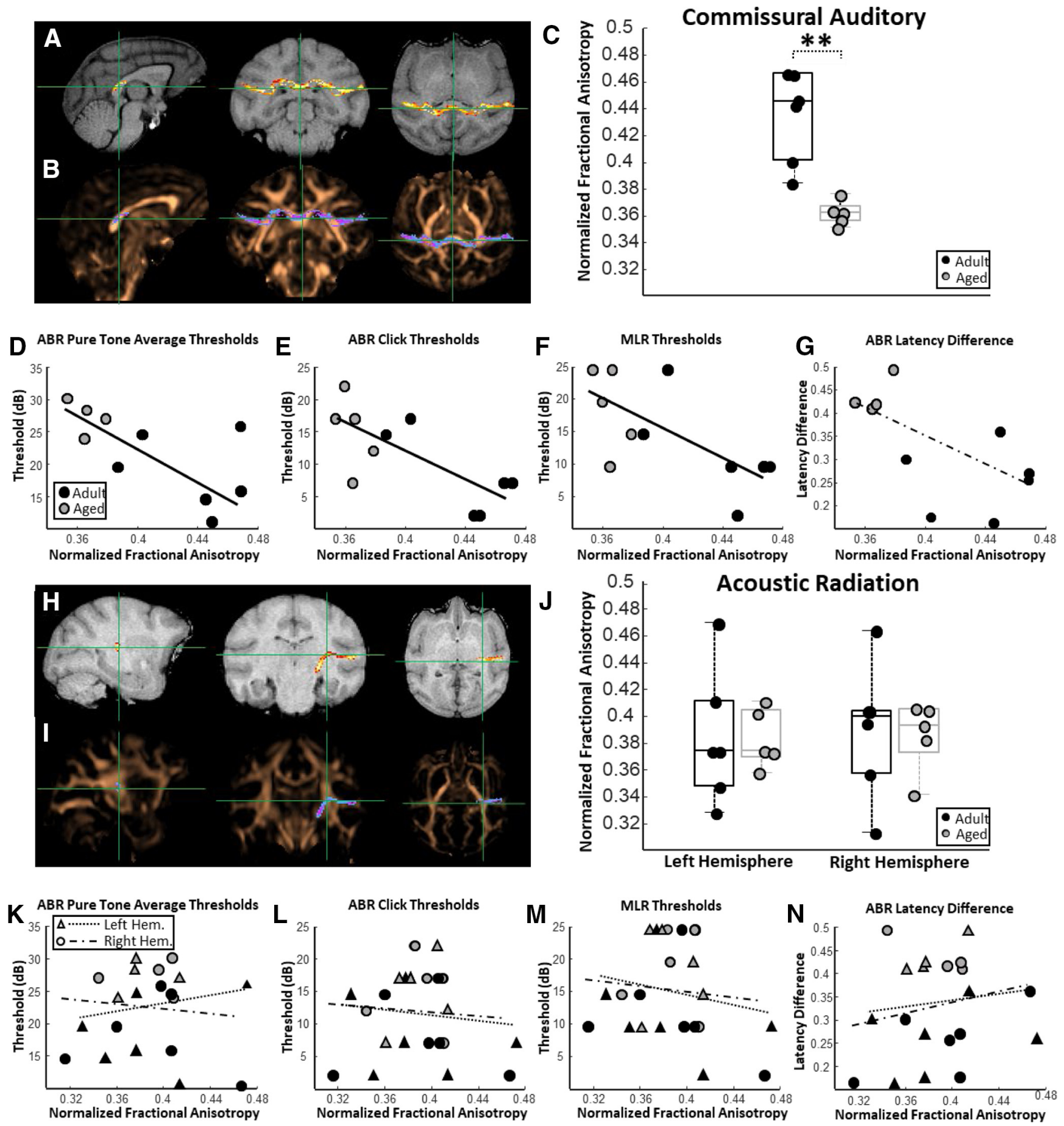

Figure 4. Relationships among auditory system white matter fractional anisotropy (FA), acoustic thresholds, and auditory temporal processing abilities. $A$, Representative probability map of voxels belonging to commissural auditory cortex connectivity overlaid on a T1-weighted MRI in sagittal, coronal, and horizontal planes. $\boldsymbol{B}$, The same probability map overlaid on a FA map pseudocolored in copper. C, Box and whisker plots of commissural auditory white matter FA estimates for each individual monkey. Boxes represent the middle 50\% of the data, and horizontal lines mark the median of each distribution. Each filled circle indicates an individual monkey, with black circles representing adult animals and gray circles representing the aged animals. Aged monkeys had significantly lower commissural auditory white matter FA compared with adult animals. $\boldsymbol{D}$, Relationship between commissural auditory white matter FA and auditory brainstem response (ABR) pure-tone average thresholds. In all scatterplots, solid trend lines represent significant relationships and dotted trend lines represent nonsignificant relationships. There was a significant negative relationship between commissural auditory white matter FA and ABR pure-tone thresholds. $E$, Relationship between commissural auditory white matter FA and middlelatency response (MLR) thresholds to click stimuli. There was a significant negative relationship between commissural auditory white matter FA and ABR click thresholds. $F$, Relationship between commissural auditory white matter FA and auditory middle-latency response (MLR) thresholds. There was a significant negative relationship between commissural auditory white matter FA and MLR thresholds. Note that in all cases lower acoustic thresholds are thought to correspond with better auditory acuity. These findings indicate that monkeys with greater FA in the corticocortical connectivity between the two auditory cortices have lower acoustic thresholds. $G$, Relationship between commissural auditory white matter FA and estimates of auditory system temporal processing abilities (ABR latency difference to pure tones presented at $50 \mathrm{vs} 20 \mathrm{~Hz}$ ). A strong yet nonsignificant negative trend was observed between commissural auditory white matter FA and ABR latency difference $(p=0.07)$. Note that lower ABR latency differences are thought to correspond with better auditory information processing. $\boldsymbol{H}$, Representative probability map of voxels belonging to acoustic thalamic radiation connectivity overlaid on a T1-weighted MRI in sagittal, coronal, and horizontal planes. I, The same probability map overlaid on a FA map pseudocolored in copper. J, Box and whisker plots of acoustic radiation FA estimates for each individual monkey separated by left and right hemisphere. Unlike the commissural auditory 
$n_{\text {aged }}=5 ; F_{(1,10)}=0.01 ; p=0.91 ;$ Fig. $\left.4 J\right)$, nor were FA estimates different between the right and left hemispheres (ANOVA; $n_{\text {adult }}=6 ; n_{\text {aged }}=5 ; F_{(1,10)}=0.05 ; p=0.83 ;$ Fig. $\left.4 J\right)$. Acoustic thalamic radiation $\mathrm{FA}$ was not associated with $\mathrm{ABR}$ pure-tone thresholds (robust regression; $n=10$; left hemisphere: $p=0.63$; $r=-0.15 ; t_{(9)}=0.49$; right hemisphere: $p=0.77 ; r=-0.15 ; t_{(9)}=$ -0.30 ; Fig. $4 K$ ), ABR click thresholds (robust regression; $n=10$; left hemisphere: $p=0.74 ; r=-0.11 ; t_{(9)}=-0.35$; right hemisphere: $p=0.82 ; r=-0.06 ; t_{(9)}=-0.24$; Fig. $4 L$ ), acoustic MLR thresholds (robust regression; $n=10$; left hemisphere: $p=0.59$; $r=-0.21 ; t_{(9)}=-0.56$; right hemisphere: $p=0.76 ; r=-0.14$; $t_{(9)}=-0.31$; Fig. $4 M$ ), or temporal-ABR latency differences (robust regression; $n=10$; left hemisphere: $p=0.77 ; \quad r=0.12$; $t_{(9)}=0.31$; right hemisphere: $p=0.59 ; r=0.19 ; t_{(9)}=0.56$; Fig. $4 N)$. Acoustic radiation FA estimates showed no relationship with temporal-VEP $\mathrm{P}_{75}$ latency differences (robust regression; $n=10$; left hemisphere: $p=0.91 ; r=0.004 ; t_{(9)}=0.12$; right hemisphere: $p=0.64 ; r=0.19 ; t_{(9)}=0.49$; data not shown).

\section{Optic radiation, but not commissural visual white matter $\mathrm{FA}$, is reduced in aged monkeys}

FA estimates were also acquired from the following two distinct visual system white matter tracts: commissural visual fibers (i.e., the posterior forceps) and the optic thalamic radiation. FA estimates from the commissural visual pathway (Fig. $5 A, B$ ) were not different between adult and aged animals (unpaired $t$ test; $n_{\text {adult }}$ $=6 ; n_{\text {aged }}=5 ; p=0.13 ; t_{(9)}=1.64 ;$ Fig. $5 C$ ). Monkeys that had the highest FA in the commissural visual pathway had significantly lower VEP latency differences, which corresponds with better visual system function (robust regression; $n=10 ; p=0.03 ; r=$ $-0.61 ; t_{(9)}=-2.66$; Fig. $5 D$ ). There was no relationship between FA estimates from the commissural visual pathway and $A B R$ PTA thresholds (robust regression; $n=10 ; p=0.61 ; r=-0.21$; $t_{(9)}=-0.54$ ) or temporal-ABR latencies (robust regression; $n=10 ; p=0.5 ; r=-0.28 ; t_{(9)}=-0.71$; data not shown).

FA estimates from the optic thalamic radiation (Fig. $5 E, F$ ) were significantly lower in aged monkeys compared with adults $\left(\right.$ ANOVA; $n_{\text {adult }}=6 ; n_{\text {aged }}=5 ; F_{(1,10)}=11.2 ; p<0.01 ;$ Fig. $\left.5 G\right)$, although there were no differences in FA between the right and left hemispheres (ANOVA; $n_{\text {adult }}=6 ; n_{\text {aged }}=5 ; F_{(1,10)}=0.37$; $p=0.55$ ). Animals that had higher FA in the right hemisphere optic thalamic radiation had significantly lower VEP latency differences (robust regression; $n=10 ; p=0.01 ; r=-0.60 ; t_{(9)}=$ -3.35 ; Fig. $5 H$ ). Left hemisphere optic thalamic radiation FA, however, was not significantly associated with VEP latency differences (robust regression; $n=10 ; p=0.58 ; r=-0.17 ; t_{(9)}=$ $-0.58)$. There was no relationship between optic radiation FA estimates and ABR PTA thresholds (robust regression; $n=10$; left hemisphere: $p=0.50 ; r=-0.26 ; t_{(9)}=-0.70$; right hemisphere: $\left.p=0.38 ; r=-0.29 ; t_{(9)}=-0.93\right)$. Similarly, there was no relationship between optic radiation FA estimates and temporalABR latency differences (robust regression; $n=10$; left hemisphere: $p=0.81 ; r=0.20 ; t_{(9)}=0.25$; right hemisphere: $p=0.60 ; r$ $=-0.20 ; t_{(9)}=-0.54$; data not shown).

$\leftarrow$

white matter, there was no impact of age on acoustic radiation FA estimates. $\boldsymbol{K}$, Relationship between acoustic radiation FA and ABR pure-tone average thresholds. Triangles represent left hemisphere acoustic radiation FA estimates, whereas circles represent FA estimates from the right hemisphere acoustic radiation. $L$, Relationship between acoustic radiation FA and ABR thresholds to click stimuli. $\boldsymbol{M}$, Relationship between acoustic radiation FA and auditory MLR thresholds. $\boldsymbol{N}$, Relationship between acoustic radiation FA and estimates of auditory system temporal processing abilities. Acoustic radiation FA was not associated with acoustic thresholds or with auditory temporal processing capacities. ${ }^{* *} p<0.01$.

\section{Discussion}

Several novel results from the present study indicate that specific corticocortical and thalamocortical white matter tracts in the auditory and visual systems undergo age-associated structural changes that contribute to deficits in sensory function within each domain. First, the FA of auditory interhemispheric corticocortical white matter was reduced in older monkeys compared with adults, whereas FA estimates from thalamocortical fibers in the acoustic thalamic radiations were not different between age groups. Conversely, the FA of visual interhemispheric corticocortical white matter was not different between age groups, whereas a significant reduction in FA was observed in thalamocortical fibers contained within the optic thalamic radiations of older monkeys relative to adults. Importantly, monkeys with higher FA in interhemispheric auditory fibers showed better auditory function, whereas higher FA estimates in both interhemispheric visual fibers and the optic radiations were associated with higher visual function. Together, these results indicate that impairments in auditory and visual function arise in part from structural changes to specific white matter projections rather than from global decreases in connectivity between sensory brain structures.

\section{Auditory temporal processing deficits arise alongside decreases in auditory sensitivity}

Two distinct facets of acoustic processing were examined in the present study: auditory sensitivity and auditory temporal information processing. The first critical finding from these evaluations was that aged monkeys had significantly greater waveform latency differences between the 50 and $20 \mathrm{~Hz}$ stimulus presentation rate conditions compared with adults in the temporal-ABR analysis. Longer latency differences in this analysis reflect poorer integration of temporal acoustic information found in complex sound stimuli (Mehraei et al., 2016, 2017). This result is consistent with observations that single neurons in the auditory cortex of aged macaques have broader spatial tuning curves (JuarezSalinas et al., 2010), and encode amplitude-modulated sound stimuli less reliably compared with younger monkeys (Overton and Recanzone, 2016; Ng and Recanzone, 2018). Another important finding facilitated by these analyses was that greater temporal-ABR latency differences occurred alongside age-associated increases in ABR thresholds. This observation indicates that older animals experience more difficulty encoding complex sound stimuli in part because of decreases in their absolute hearing sensitivity. Since threshold-ABR measures largely reflect cochlear function (Fowler et al., 2010; Engle et al., 2013), these findings indicate that the trajectory of function that the central auditory system takes across the lifespan is influenced by peripheral hearing deficits. Note, however, that structural and functional changes to the central auditory system also arise somewhat independently of peripheral changes. For example, older humans with clinically normal audiometric thresholds experience difficulties in understanding temporally compressed human speech relative to younger adults (Peelle et al., 2010; Peelle and Wingfield, 2016).

\section{Visual function is not strongly associated with auditory function}

Visual sensory function was not statistically different between the adult and aged animals, although there was a clear trend in older animals to have poorer function. The temporal-VEP analysis was designed to estimate visual processing speed, a 

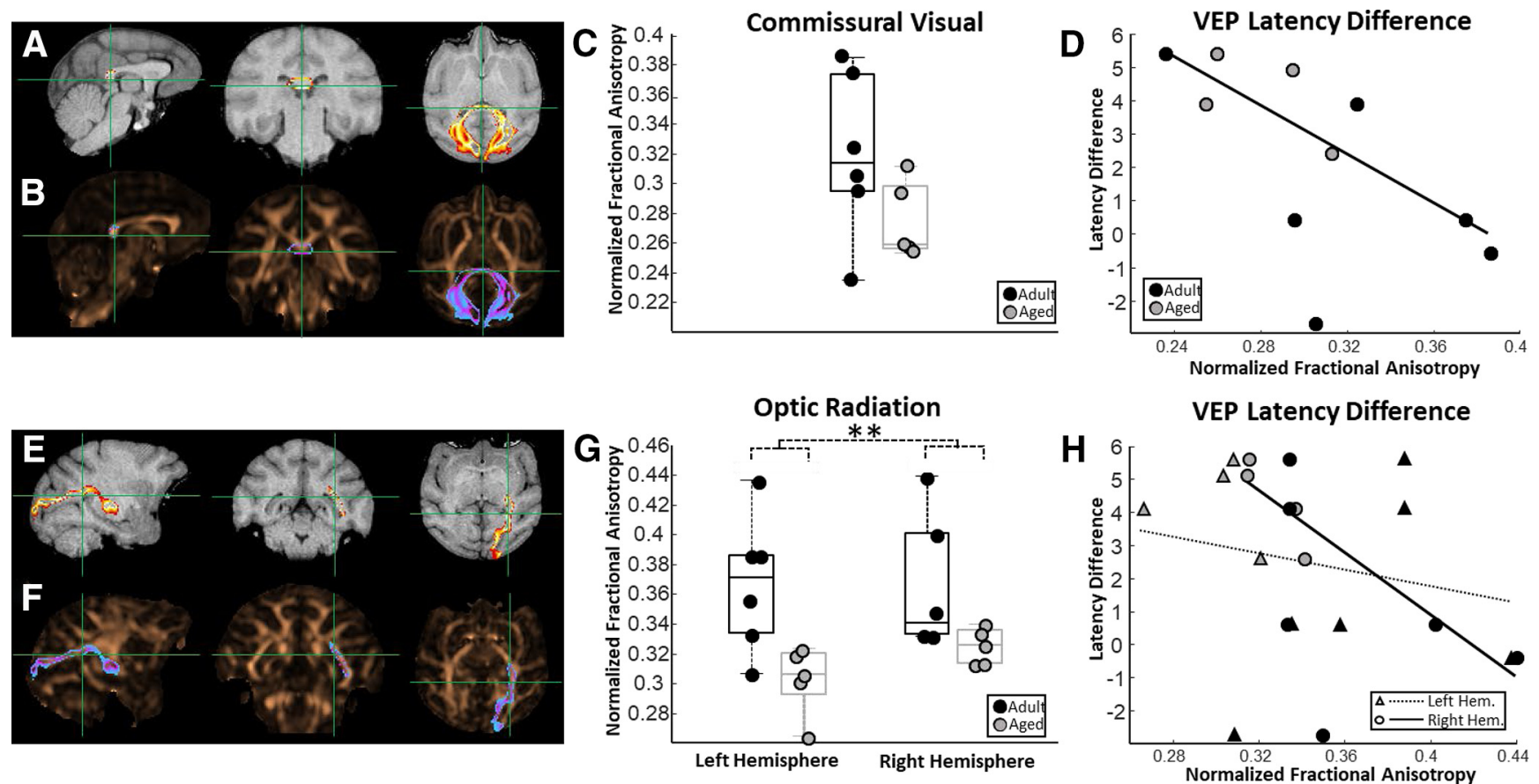

Figure 5. Relationships between visual system white matter fractional anisotropy (FA) and estimates of visual system processing abilities. $\boldsymbol{A}$, Representative probability map of voxels belonging to commissural visual cortex connectivity overlaid on a T1-weighted MRI in sagittal, coronal, and horizontal planes. $\boldsymbol{B}$, The same probability map overlaid on a FA map pseudocolored in copper. C, Box and whisker plots of commissural visual white matter FA estimates for each individual monkey. Boxes represent the middle 50\% of the data, and horizontal lines mark the median of each distribution. Each filled circle indicates an individual monkey, with black circles representing adult animals and gray circles representing the aged. Commissural visual white matter FA was not different between adult and aged monkeys. D, Relationship between commissural visual white matter FA and estimates of visual system function (visual evoked potential (VEP) latency differences to light presented at 2 and $1 \mathrm{~Hz}$ ). In all scatterplots, solid trend lines represent significant relationships and dotted trend lines represent nonsignificant relationships. There was a significant negative relationship between commissural visual white matter FA and VEP latency differences. Note that lower VEP latency differences are thought to correspond to better visual function. $\boldsymbol{E}$, Representative probability map of voxels belonging to optic thalamic radiation connectivity overlaid on a T1-weighted MRl. $\boldsymbol{F}$, The same probability map overlaid on a FA map pseudocolored in copper. G, Box and whisker plots of optic radiation FA estimates for each individual monkey separated by left and right hemisphere. Aged monkeys had significantly lower optic radiation FA estimates than did the adult animals. $\boldsymbol{H}$, Relationship between optic radiation FA and estimates of visual system processing abilities. Triangles represent left hemisphere optic radiation FA estimates, whereas circles represent FA estimates from the right hemisphere optic radiation. A significant negative relationship was observed between right hemisphere optic radiation FA and visual system function. ${ }^{* *} p<0.01$.

component of visual function that reliably worsens in older humans and is thought to largely reflect impairments in central visual structures downstream from the retina (Birren and Fisher, 1991; Owsley, 2011, 2016). Whether other aspects of visual sensory function also worsen in aged monkeys cannot be determined from the data in the present study, and a critical future direction will be to collect more comprehensive assessments of visual function in aged monkeys. Furthermore, no measure of auditory function was significantly associated with VEP $\mathrm{P}_{75}$ latency differences, although subtle trends were observed such that individual animals with better visual function also tended to have better auditory function. Although a larger sample of aging monkeys is necessary for a definitive conclusion, these results suggest that age-associated alterations in auditory and visual sensory function arise with some degree of independence from one another in macaques.

\section{Tract-specific reductions in sensory white matter FA}

FA estimates were extracted from both auditory and visual system corticocortical and thalamocortical white matter tracts within the same group of animals that underwent electrophysiological assessments of auditory and visual function. Monkeys with higher auditory system white matter FA tended to have higher visual system white matter FA. This relationship was not because of general reductions in FA across the two sensory systems, but rather from age-associated FA reductions in specific white matter tracts within each sensory system. FA estimates from commissural fibers connecting the auditory cortices in each hemisphere were significantly lower in the older monkeys compared with the adult animals, whereas FA estimates of commissural fibers connecting the two visual cortices were not different between the adult and aged animals. The opposite pattern was observed in the thalamic radiations of each sensory system such that FA estimates from the acoustic thalamic radiations were not different between the adult and aged animals, whereas the aged monkeys had significantly lower FA in their optic radiations. Together, these results indicate that age-associated changes in sensory system white matter are not because of general factors that might impact multiple pathways in tandem, but rather because of factors inherent to the anatomic location of a particular fiber tract within the brain.

While it is not certain what regional factors impact the structure and function of individual white matter tracts uniquely, several ideas might be considered. One possibility is that the proximity of white matter to major brain vasculature dictates the structure and function of a tract across the lifespan. This hypothesis is supported by observations that neurovascular dysfunction commonly arises in older individuals to negatively impact brain function (Nelson et al., 2016; Sweeney et al., 2018); furthermore, occlusions of distinct cerebral arteries result in unique patterns of cognitive impairment in older individuals (Fabiani et al., 2014). Another idea is that functional and anatomic differences between the visual and auditory systems at their subthalamocortical levels underlie differences in how the normative aging 
process impacts each system. In the auditory system, information is relayed from the cochlea through multiple brainstem and midbrain processing centers before arriving at the medial geniculate-auditory cortex pathway (Harrison and Howe, 1974; Ehret and Romand, 1997; Jones, 2003). Conversely, the majority of visual information leaving the retina is sent directly to the lateral geniculate-visual cortex pathway and never enters the midbrain or brainstem (Prasad and Galetta, 2011; De Moraes, 2013). Perhaps the anatomic arrangement of the visual system reflects a stronger dependency on thalamocortical interactions in representing sensory information compared with the auditory system. The present data support this hypothesis since FA estimates from the acoustic thalamic radiations showed no relationship with any measure of acoustic function, whereas monkeys with higher FA in the optic radiations displayed better visual function. Other regional factors that could influence a specific white matter tract uniquely from others include genetic and functional differences between neurons and glial cells contained within distinct brain areas (Ma et al., 2013; Khakh and Sofroniew, 2015; De Biase and Bonci, 2018), and regional differences in patterns of age-associated hyperexcitability/excitotoxicity (Yassa et al., 2011; Thomé et al., 2016; Recanzone, 2018).

A previous study from our group used these same monkeys to demonstrate FA reductions in white matter tracts associated with medial temporal lobe brain structures, but not in white matter tracts connecting frontal cortical brain regions (Gray et al., 2019). Specifically, higher FA in the fimbria-fornix and hippocampal commissure was related to better performance on medial temporal lobe-dependent tasks, but not behaviors dependent on frontal lobe structures. These data were interpreted to reflect differential alterations in temporal versus frontal lobe structure and function across the lifespan. The present data support the idea that white matter contained within different lobes of the brain are uniquely vulnerable to structural changes that arise because of normative brain aging. A fruitful future direction will be to collect regional estimates of gray and white matter structure alongside regional estimates of excitotoxicity, neurovascular function, and glial cell function within the same set of aging animals. Such data might shift the focus of brain aging research toward understanding local adaptation in brain function to vulnerabilities with relatively local influence in the brain (Gray and Barnes, 2015).

\section{Conclusions}

The results from this study indicate that aged monkeys express poorer auditory and visual system function and lower FA in specific sensory thalamocortical and corticocortical white matter tracts compared with adult monkeys. These findings indicate that age-related declines in sensory processing do not arise from general decreases in connectivity between sensory brain regions, but rather from factors that impact specific white matter tracts. It will be important moving forward to design experiments that acquire multiple estimates of sensory and cognitive system structure and function across multiple domains within the same individuals. These efforts will continue to elucidate the neurobiological mechanisms responsible for the unique patterns of sensory and cognitive decline observed in healthy older individuals, and will aid in designing individualized intervention strategies that optimally correct and maintain brain function across the lifespan.

\section{References}

Abe O, Yamasue H, Aoki S, Suga M, Yamada H, Kasai K, Masutani Y, Kato N, Kato N, Ohtomo K (2008) Aging in the CNS: comparison of gray/ white matter volume and diffusion tensor data. Neurobiol Aging 29:102116.

Allen AR, Starr A (1978) Auditory brain stem potentials in monkey (M. mulatta) and man. Electroencephalogr Clin Neurophysiol 45:53-63.

Andersen GJ (2012) Aging and vision: changes in function and performance from optics to perception. Wiley Interdiscip Rev Cogn Sci 3:403-410.

Andersson JLR, Sotiropoulos SN (2015) Non-parametric representation and prediction of single- and multi-shell diffusion-weighted MRI data using Gaussian processes. Neuroimage 122:166-176.

Bamiou D-E, Sisodiya S, Musiek FE, Luxon LM (2007) The role of the interhemispheric pathway in hearing. Brain Res Rev 56:170-182.

Bennett IJ, Madden DJ (2014) Disconnected aging: cerebral white matter integrity and age-related differences in cognition. Neuroscience 276:187-205.

Bennett IJ, Madden DJ, Vaidya CJ, Howard DV, Howard JH Jr (2010) Agerelated differences in multiple measures of white matter integrity: a diffusion tensor imaging study of healthy aging. Hum Brain Mapp 31:378-390.

Berman JI, Lanza MR, Blaskey L, Edgar JC, Roberts TPL (2013) High angular resolution diffusion imaging probabilistic tractography of the auditory radiation. AJNR Am J Neuroradiol 34:1573-1578.

Birren JE, Fisher LM (1991) Aging and slowing of behavior: consequences for cognition and survival. Neb Symp Motiv Neb Symp Motiv 39:1-37.

Bourne RRA, Flaxman SR, Braithwaite T, Cicinelli MV, Das A, Jonas JB, Keeffe J, Kempen JH, Leasher J, Limburg H, Naidoo K, Pesudovs K, Resnikoff S, Silvester A, Stevens GA, Tahhan N, Wong TY, Taylor HR (2017) Magnitude, temporal trends, and projections of the global prevalence of blindness and distance and near vision impairment: a systematic review and meta-analysis. Lancet Glob Health 5:e888-e897.

Brown RL, Barrett AE (2011) Visual impairment and quality of life among older adults: an examination of explanations for the relationship. J Gerontol B Psychol Sci Soc Sci 66:364-373.

Burke SN, Wallace JL, Hartzell AL, Nematollahi S, Plange K, Barnes CA (2011) Age-associated deficits in pattern separation functions of the perirhinal cortex: a cross-species consensus. Behav Neurosci 125:836-847.

Burke SN, Thome A, Plange K, Engle JR, Trouard TP, Gothard KM, Barnes CA (2014) Orbitofrontal cortex volume in area 11/13 predicts reward devaluation, but not reversal learning performance, in young and aged monkeys. J Neurosci 34:9905-9916.

Campo P, Morata TC, Hong O (2013) Chemical exposure and hearing loss. Dis Mon 59:119-138.

Chang Y, Lee S-H, Lee Y-J, Hwang M-J, Bae S-J, Kim M-N, Lee J, Woo S, Lee H, Kang D-S (2004) Auditory neural pathway evaluation on sensorineural hearing loss using diffusion tensor imaging. Neuroreport 15:1699-1703.

Comrie AE, Gray DT, Smith AC, Barnes CA (2018) Different macaque models of cognitive aging exhibit task-dependent behavioral disparities. Behav Brain Res 344:110-119.

De Biase LM, Bonci A (2018) Region-specific phenotypes of microglia: the role of local regulatory cues. Neuroscientist 25:314-333.

De Moraes CG (2013) Anatomy of the visual pathways. J Glaucoma 22:S2-S7.

Ehret G, Romand R (1997) The central auditory system. Oxford, UK: Oxford UP.

Engle JR, Tinling S, Recanzone GH (2013) Age-related hearing loss in rhesus monkeys is correlated with cochlear histopathologies. PLoS One 8:e55092.

Essen DV, Newsome WT, Bixby JL (1982) The pattern of interhemispheric connections and its relationship to extrastriate visual areas in the macaque monkey. J Neurosci 2:265-283.

Fabiani M, Low KA, Tan C-H, Zimmerman B, Fletcher MA, SchneiderGarces N, Maclin EL, Chiarelli AM, Sutton BP, Gratton G (2014) Taking the pulse of aging: mapping pulse pressure and elasticity in cerebral arteries with optical methods. Psychophysiology 51:1072-1088.

Farnum A, Pelled G (2020) New vision for visual prostheses. Front Neurosci $14: 36$

Fowler CG, Chiasson KB, Leslie TH, Thomas D, Beasley TM, Kemnitz JW, Weindruch R (2010) Auditory function in rhesus monkeys: effects of aging and caloric restriction in the Wisconsin monkeys five years later. Hear Res 261:75-81.

Fransen E, Topsakal V, Hendrickx J-J, Van Laer L, Huyghe JR, Van Eyken E, Lemkens N, Hannula S, Mäki-Torkko E, Jensen M, Demeester K, Tropitzsch A, Bonaconsa A, Mazzoli M, Espeso A, Verbruggen K, Huyghe J, Huygen PLM, Kunst S, Manninen M, et al. (2008) Occupational noise, smoking, and a high body mass index are risk factors for age-related hearing impairment and moderate alcohol consumption 
is protective: a European population-based multicenter study. J Assoc Res Otolaryngol. 9:264-276.

Gheorghe A, Mahdi L, Musat O (2015) Age-related macular degeneration. Rom J Ophthalmol 59:74-77.

Gopinath B, Rochtchina E, Wang JJ, Schneider J, Leeder SR, Mitchell P (2009) Prevalence of age-related hearing loss in older adults: blue mountains study. Arch Intern Med 169:415-418.

Gray DT, Barnes CA (2015) Distinguishing adaptive plasticity from vulnerability in the aging hippocampus. Neuroscience 309:17-28.

Gray DT, Barnes CA (2019) Experiments in macaque monkeys provide critical insights into age-associated changes in cognitive and sensory function. Proc Natl Acad Sci U S A 116:26247-26254.

Gray DT, Smith AC, Burke SN, Gazzaley A, Barnes CA (2017) Attentional updating and monitoring and affective shifting are impacted independently by aging in macaque monkeys. Behav Brain Res 322:329-338.

Gray DT, Umapathy L, Burke SN, Trouard TP, Barnes CA (2018) Tract-specific white matter correlates of age-related reward devaluation deficits in macaque monkeys. J Neuroimaging Psychiatry Neurol 3:13-26.

Gray DT, Umapathy L, De La Peña NM, Burke SN, Engle JR, Trouard TP, Barnes CA (2019) Auditory processing deficits are selectively associated with medial temporal lobe mnemonic function and white matter integrity in aging macaques. Cereb Cortex 30:2789-2803.

Greve DN, Fischl B (2009) Accurate and robust brain image alignment using boundary-based registration. Neuroimage 48:63-72.

Hackett TA, Stepniewska I, Kaas JH (1998) Thalamocortical connections of the parabelt auditory cortex in macaque monkeys. J Comp Neurol 400:271-286.

Hackett TA, Preuss TM, Kaas JH (2001) Architectonic identification of the core region in auditory cortex of macaques, chimpanzees, and humans. J Comp Neurol 441:197-222.

Hara Y, Rapp PR, Morrison JH (2012) Neuronal and morphological bases of cognitive decline in aged rhesus monkeys. Age 34:1051-1073.

Harrison JM, Howe ME (1974) 283-336. Anatomy of the afferent auditory nervous system of mammals. In: Auditory system: handbook of sensory physiology. Berlin: Springer.

Helzner EP, Patel AS, Pratt S, Sutton-Tyrrell K, Cauley JA, Talbott E, Kenyon E, Harris TB, Satterfield S, Ding J, Newman AB (2011) Hearing sensitivity in older adults: associations with cardiovascular risk factors in the health, aging and body composition study. J Am Geriatr Soc 59:972-979.

Husain FT, Medina RE, Davis CW, Szymko-Bennett Y, Simonyan K, Pajor NM, Horwitz B (2011) Neuroanatomical changes due to hearing loss and chronic tinnitus: a combined VBM and DTI study. Brain Res 1369:74-88.

Jones EG (2003) Chemically defined parallel pathways in the monkey auditory system. Ann N Y Acad Sci 999:218-233.

Jones EG (2009) Synchrony in the interconnected circuitry of the thalamus and cerebral cortex. Ann N Y Acad Sci 1157:10-23.

Juarez-Salinas DL, Engle JR, Navarro XO, Recanzone GH (2010) Hierarchical and serial processing in the spatial auditory cortical pathway is degraded by natural aging. J Neurosci 30:14795-14804.

Kaas JH, Hackett TA (2000) Subdivisions of auditory cortex and processing streams in primates. Proc Natl Acad Sci U S A 97:11793-11799.

Khakh BS, Sofroniew MV (2015) Diversity of astrocyte functions and phenotypes in neural circuits. Nat Neurosci 18:942-952.

Lim LS, Mitchell P, Seddon JM, Holz FG, Wong TY (2012) Age-related macular degeneration. The Lancet 379:1728-1738.

Lin FR, Ferrucci L, An Y, Goh JO, Doshi J, Metter EJ, Davatzikos C, Kraut MA, Resnick SM (2014) Association of hearing impairment with brain volume changes in older adults. NeuroImage 90:84-92.

Lockhart SN, DeCarli C (2014) Structural imaging measures of brain aging. Neuropsychol Rev 24:271-289.

Ma T, Wang C, Wang L, Zhou X, Tian M, Zhang Q, Zhang Y, Li J, Liu Z, Cai Y, Liu F, You Y, Chen C, Campbell K, Song H, Ma L, Rubenstein JL, Yang Z (2013) Subcortical origins of human and monkey neocortical interneurons. Nat Neurosci 16:1588-1597.

Madden DJ, Whiting WL, Huettel SA, White LE, MacFall JR, Provenzale JM (2004) Diffusion tensor imaging of adult age differences in cerebral white matter: relation to response time. Neuroimage 21:1174-1181.

Makris N, Papadimitriou GM, van der Kouwe A, Kennedy DN, Hodge SM, Dale AM, Benner T, Wald LL, Wu O, Tuch DS, Caviness VS, Moore TL, Killiany RJ, Moss MB, Rosene DL (2007) Frontal connections and cognitive changes in normal aging rhesus monkeys: a DTI study. Neurobiol Aging 28:1556-1567.
Manjón JV, Coupé P, Concha L, Buades A, Collins DL, Robles M (2013) Diffusion weighted image denoising using overcomplete local PCA. PLoS One 8:e73021.

Mehraei G, Hickox AE, Bharadwaj HM, Goldberg H, Verhulst S, Liberman MC, Shinn-Cunningham BG (2016) Auditory brainstem response latency in noise as a marker of cochlear synaptopathy. J Neurosci 36:3755-3764.

Mehraei G, Gallardo AP, Shinn-Cunningham BG, Dau T (2017) Auditory brainstem response latency in forward masking, a marker of sensory deficits in listeners with normal hearing thresholds. Hear Res 346:34-44.

Mitchell P, Gopinath B, McMahon CM, Rochtchina E, Wang JJ, Boyages SC, Leeder SR (2009) Relationship of type 2 diabetes to the prevalence, incidence and progression of age-related hearing loss. Diabet Med J Br Diabet Assoc 26:483-488.

Nelson AR, Sweeney MD, Sagare AP, Zlokovic BV (2016) Neurovascular dysfunction and neurodegeneration in dementia and Alzheimer's disease. Biochim Biophys Acta 1862:887-900.

Ng C-W, Recanzone GH (2018) Age-related changes in temporal processing of rapidly-presented sound sequences in the macaque auditory cortex. Cereb Cortex 28:3775-3796.

Ng C-W, Navarro X, Engle JR, Recanzone GH (2015) Age-related changes of auditory brainstem responses in nonhuman primates. J Neurophysiol 114:455-467.

Overton JA, Recanzone GH (2016) Effects of aging on the response of single neurons to amplitude-modulated noise in primary auditory cortex of rhesus macaque. J Neurophysiol 115:2911-2923.

Owsley C (2011) Aging and vision. Vision Res 51:1610-1622.

Owsley C (2016) Vision and aging. Annu Rev Vis Sci 2:255-271.

Peelle JE, Wingfield A (2016) The neural consequences of age-related hearing loss. Trends Neurosci 39:486-497.

Peelle JE, Troiani V, Wingfield A, Grossman M (2010) Neural processing during older adults' comprehension of spoken sentences: age differences in resource allocation and connectivity. Cereb Cortex 20:773-782.

Petrides M, Pandya DN (2003) Comparative architectonic analysis of the human and the macaque frontal cortex. In: Handbook of neuropsychology: plasticity and rehabilitation. (Boller F, Grafman J, Robertson IH, eds), pp 17-58. Amsterdam: Elsevier.

Plontke SK, Götze G, Rahne T, Liebau A (2017) Intracochlear drug delivery in combination with cochlear implants: current aspects. HNO 65:19-28.

Prasad S, Galetta SL (2011) Anatomy and physiology of the afferent visual system. In: Handbook of clinical neurology: neuro-ophthalmology (Kennard C, Leigh RJ, eds), pp 3-19. Amsterdam: Elsevier.

Profant O, Škoch A, Balogová Z, Tintěra J, Hlinka J, Syka J (2014) Diffusion tensor imaging and MR morphometry of the central auditory pathway and auditory cortex in aging. Neuroscience 260:87-97.

Recanzone G (2018) The effects of aging on auditory cortical function. Hear Res 366:99-105.

Ryan AF (2000) Protection of auditory receptors and neurons: evidence for interactive damage. Proc Natl Acad Sci U S A 97:6939-6940.

Schmiedt RA (2010) The physiology of cochlear presbycusis. In: The aging auditory system: Springer handbook of auditory research, pp 9-38. New York: Springer.

Sherman SM (2017) Functioning of circuits connecting thalamus and cortex. Compr Physiol 7:713-739.

Sweeney MD, Kisler K, Montagne A, Toga AW, Zlokovic BV (2018) The role of brain vasculature in neurodegenerative disorders. Nat Neurosci 21:1318-1331.

Thomé A, Gray DT, Erickson CA, Lipa P, Barnes CA (2016) Memory impairment in aged primates is associated with region-specific network dysfunction. Mol Psychiatry 21:1257-1262.

Tustison NJ, Avants BB, Cook PA, Zheng Y, Egan A, Yushkevich PA, Gee JC (2010) N4ITK: improved N3 Bias correction. IEEE Trans Med Imaging 29:1310-1320.

Wattamwar K, Qian ZJ, Otter J, Leskowitz MJ, Caruana FF, Siedlecki B, Spitzer JB, Lalwani AK (2017) Increases in the rate of age-related hearing loss in the older old. JAMA Otolaryngol Neck Surg 143:41-45.

Yam JCS, Kwok AKH (2014) Ultraviolet light and ocular diseases. Int Ophthalmol 34:383-400.

Yassa MA, Lacy JW, Stark SM, Albert MS, Gallagher M, Stark CEL (2011) Pattern separation deficits associated with increased hippocampal CA3 and dentate gyrus activity in nondemented older adults. Hippocampus 21:968979. 